Article

\title{
On Generalized Nonexpansive Maps in Banach Spaces
}

\author{
Kifayat Ullah ${ }^{1}\left(\mathbb{D}\right.$, Junaid Ahmad ${ }^{1, *(1)}$ and Manuel de la Sen ${ }^{2}$ (I) \\ 1 Department of Mathematics, University of Science and Technology, \\ Bannu 28100, Khyber Pakhtunkhwa, Pakistan; kifayatmath@yahoo.com \\ 2 Institute of Research and Development of Processes, University of the Basque Country, Campus of Leioa \\ (Bizkaia), P.O. Box 644- Bilbao, Barrio Sarriena, 48940 Leioa, Spain; manuel.delasen@ehu.eus \\ * Correspondence: ahmadjunaid436@gmail.com
}

Received: 14 May 2020; Accepted: 30 June 2020; Published: 3 July 2020

\begin{abstract}
We introduce a very general class of generalized non-expansive maps. This new class of maps properly includes the class of Suzuki non-expansive maps, Reich-Suzuki type non-expansive maps, and generalized $\alpha$-non-expansive maps. We establish some basic properties and demiclosed principle for this class of maps. After this, we establish existence and convergence results for this class of maps in the context of uniformly convex Banach spaces and compare several well known iterative algorithms.
\end{abstract}

Keywords: generalized non-expansive map; demiclosed principle; uniformly convex Banach space; rate of convergence; Banach space

\section{Introduction}

Let $X$ be a Banach space, $E$ be a nonempty subset of $X$ and $P: E \rightarrow E$ be a selfmap. An element $z \in E$ is called a fixed point of $P$ if $z=P(z)$. From now on, we will denote the set of all fixed points of $P$ by the notation $F(P)$. The map $P$ is called non-expansive if $\|P(a)-P(b)\| \leq\|a-b\|$ for all $a, b \in E$ and it is called quasi-non-expansive if $\|P(z)-P(a)\| \leq\|z-a\|$ for all $a \in E$ and $z \in F(P)$. In 1965, Kirk [1], Browder [2], and Gohde [3] independently proved that every non-expansive map has a fixed point if $E$ is closed bounded convex and $X$ is uniformly convex. Fixed point theory of non-expansive and generalized non-expansive maps in an appropriate domain is an important research area on its own and has applications in image recovery and signal processing (see, e.g., [4-8] and references cited therein).

In 2008, Suzuki [9] suggested a weaker notion of non-expansive maps as follows:

Definition 1. [9] A selfmap P on a subset $E$ of a Banach space is said to satisfy condition (C) (or said to be Suzuki non-expansive) if for each two elements $a, b \in E$,

$$
\frac{1}{2}\|a-P(a)\| \leq\|a-b\| \Rightarrow\|P(a)-P(b)\| \leq\|a-b\| .
$$

Remark 1. It is clear that every non-expansive map is Suzuki non-expansive. However, an example in [9] shows that there exists maps which are Suzuki non-expansive but not non-expansive.

In 2011, Aoyama and Kohsaka [10] proposed the class of $\alpha$-non-expansive maps as follows: 
Definition 2. [10] A selfmap P on a subset E of a Banach space is said to be $\alpha$-non-expansive if one can find a real number $\alpha \in[0,1)$ such that for each two elements $a, b \in E$,

$$
\|P(a)-P(b)\|^{2} \leq \alpha\|a-P(b)\|^{2}+\alpha\|b-P(a)\|^{2}+(1-2 \alpha)\|a-b\|^{2} .
$$

In 2017, Pant and Shukla [11] proposed the class of generalized $\alpha$-non-expansive maps as follows:

Definition 3. [11] A selfmap P on a subset E of a Banach space is said to be generalized $\alpha$-non-expansive if one can find a real number $\alpha \in[0,1)$ such that for each two elements $a, b \in E$,

$$
\begin{gathered}
\frac{1}{2}\|a-P(a)\| \leq\|a-b\| \Rightarrow \\
\|P(a)-P(b)\| \leq \alpha\|b-P(a)\|+\alpha\|a-P(b)\|+(1-2 \alpha)\|a-b\| .
\end{gathered}
$$

Remark 2. It is clear that every Suzuki non-expansive map is generalized 0-non-expansive. However, an example in [11] shows that there exist maps which are generalized $\alpha$-non-expansive but not Suzuki non-expansive.

In 2019, Pant and Pandey [12] proposed the class of Reich-Suzuki type non-expansive maps as follows:

Definition 4. [12] A selfmap P on a subset E of a Banach space is said to be $\beta$-Reich-Suzuki type non-expansive if one can find a real number $\beta \in[0,1)$ such that for each two elements $a, b \in E$,

$$
\begin{gathered}
\frac{1}{2}\|a-P(a)\| \leq\|a-b\| \Rightarrow \\
\|P(a)-P(b)\| \leq \beta\|a-P(a)\|+\beta\|b-P(b)\|+(1-2 \beta)\|a-b\| .
\end{gathered}
$$

Remark 3. It is clear that every Suzuki non-expansive map is 0-Reich-Suzuki type non-expansive. However an example in [12] shows that there exists maps which are $\beta$-Reich-Suzuki type non-expansive but not Suzuki non-expansive.

Motivated by the above definitions, in this paper, we introduce a new class of generalized non-expansive maps which is properly larger than the the class of Suzuki non-expansive maps, generalized $\alpha$-non-expansive maps and Reich-Suzuki type non-expansive maps. We also establish some basic results for this class. In this way, we improve and extend many well known corresponding results of the metric fixed point theory.

\section{Preliminaries}

A Banach space $X$ is called uniformly convex [13] if, for any real number $\varepsilon \in[0,1)$, one can find a real number $\delta \in(0, \infty)$ such that, $\frac{\|a+b\|}{2} \leq(1-\delta)$, whenever $\|a\| \leq 1,\|b\| \leq 1$ and $\|a-b\| \geq \varepsilon$ for each $a, b \in E$. $X$ is called strict convex if, for any $a, b \in X$ satisfying $\|a\|=\|b\|=1$ and $a \neq b$, it follows that $\|a+b\|<2$.

A Banach space $X$ is said to satisfy Opial condition [14], if, for every weakly convergent sequence $\left\{a_{n}\right\} \subseteq X$ with weak limit say $w \in X$, it follows that

$$
\liminf _{m \rightarrow \infty}\left\|a_{m}-w\right\|<\liminf _{m \rightarrow \infty}\left\|a_{m}-w^{\prime}\right\| \text { for all } w^{\prime} \in X-\{w\}
$$

Let $E$ be a nonempty subset of a Banach space $X$ and $\left\{a_{m}\right\}$ a bounded sequence in $X$. For each $a \in E$, define:

- $\quad$ asymptotic radius of $\left\{a_{m}\right\}$ at $a$ by $A_{r}\left(a,\left\{a_{m}\right\}\right):=\limsup _{m \rightarrow \infty}\left\|a-a_{m}\right\|$; 
- $\quad$ asymptotic radius of $\left\{a_{m}\right\}$ relative to $E$ by $A_{r}\left(E,\left\{a_{m}\right\}\right)=\inf \left\{A_{r}\left(a,\left\{a_{m}\right\}\right): a \in E\right\}$;

- $\quad$ asymptotic center of $\left\{a_{m}\right\}$ relative to $E$ by $A_{c}\left(E,\left\{a_{m}\right\}\right)=\left\{a \in E: A_{r}\left(a,\left\{a_{m}\right\}\right)=A_{r}\left(E,\left\{a_{m}\right\}\right)\right\}$.

When the space $X$ is uniformly convex [13], then the set $A_{\mathcal{c}}\left(E,\left\{a_{m}\right\}\right)$ is always singleton. Notice also that the set $A_{c}\left(E,\left\{a_{m}\right\}\right)$ is convex as well as nonempty provided that $E$ is weakly compact convex (see, e.g., $[15,16])$.

The following result is a characterization of uniform convexity, which can be found in [17].

Lemma 1. Let $X$ be a uniformly convex Banach space and $0<s \leq k_{m} \leq t<1$ for every $m \geq 1$. If $\left\{y_{m}\right\}$ and $\left\{z_{m}\right\}$ are two sequences in $X$ such that $\limsup _{m \rightarrow \infty}\left\|y_{m}\right\| \leq \gamma, \limsup _{m \rightarrow \infty}\left\|z_{m}\right\| \leq \gamma$ and $\lim _{m \rightarrow \infty}\left\|k_{m} y_{m}+\left(1-k_{m}\right) z_{m}\right\|=\gamma$ for some $\gamma \geq 0$, then $\lim _{m \rightarrow \infty}\left\|y_{m}-z_{m}\right\|=0$.

\section{Generalized $(\alpha, \beta)$-Non-Expansive Mappings}

Inspired by above and [18], we suggest a two parametric class of nonlinear maps.

Definition 5. A selfmap $P$ on a subset $E$ of a Banach space is said to be generalized $(\alpha, \beta)$-non-expansive if there exists real numbers $\alpha, \beta \in \mathbb{R}^{+}$satisfying $\alpha+\beta<1$ such that, for all $a, b \in E$,

$$
\begin{aligned}
\frac{1}{2}\|a-P(a)\| \leq\|a-b\| & \Rightarrow\|P(a)-P(b)\| \leq \alpha\|a-P(b)\|+\alpha\|b-P(a)\|+\beta\|a-P(a)\| \\
& +\beta\|b-P(b)\|+(1-2 \alpha-2 \beta)\|a-b\| .
\end{aligned}
$$

The following proposition gives many examples of generalized $(\alpha, \beta)$-non-expansive maps.

Proposition 1. Let P be a selfmap on a subset E of a Banach space. Then, the following hold:

(i) If $P$ is Suzuki non-expansive, then $P$ is generalized $(0,0)$-non-expansive.

(ii) If $P$ is generalized $\alpha$-non-expansive, then $P$ is generalized $(\alpha, 0)$-non-expansive.

(iii) If $P$ is $\beta$-Reich-Suzuki type non-expansive, then $P$ is generalized $(0, \beta)$-non-expansive.

We prove a key lemma.

Lemma 2. Let $P$ be a selfmap on a subset $E$ of a Banach space. If $P$ is generalized $(\alpha, \beta)$-non-expansive with a fixed point $z$, then $P$ is quasi-non-expansive.

Proof. Let $z \in F(P)$. Since $\frac{1}{2}\|z-P(z)\|=0 \leq\|a-z\|$, we have

$$
\begin{aligned}
\|z-P(a)\| & =\|P(z)-P(a)\| \\
& \leq \alpha\|a-P(z)\|+\alpha\|z-P(a)\|+\beta\|z-P(z)\|+\beta\|a-P(a)\|+(1-2 \alpha-2 \beta)\|a-z\| \\
& =\alpha\|a-z\|+\alpha\|z-P(a)\|+\beta\|a-P(a)\|+(1-2 \alpha-2 \beta)\|a-z\| \\
& \leq \alpha\|a-z\|+\alpha\|z-P(a)\|+\beta(\|a-z\|+\|z-P(a)\|)+(1-2 \alpha-2 \beta)\|a-z\| \\
& =\alpha\|z-P(a)\|+\beta\|z-P(a)\|+(1-\alpha-\beta)\|a-z\| .
\end{aligned}
$$

It follows that

$$
(1-\alpha-\beta)\|z-P(a)\| \leq(1-\alpha-\beta)\|z-a\| .
$$

Since $(1-\alpha-\beta)>0$, we obtain our desired result.

From Lemma 2, we obtain the following.

Lemma 3. Let $P$ be a selmap on a subset $E$ of a Banach space $X$. If $P$ is generalized $(\alpha, \beta)$-non-expansive, then the set $F(P)$ is closed. Moreover, $F(P)$ is convex provided that $E$ is convex and $X$ is strictly convex. 
We now prove the following facts.

Lemma 4. Let $P$ be a selfmap on a subset $E$ of a Banach space. If $P$ is generalized $(\alpha, \beta)$-non-expansive, then for each $a, b \in E$ :

(i) $\left\|P(a)-P^{2}(a)\right\| \leq\|a-P(a)\|$.

(ii) Either $\frac{1}{2}\|a-P(a)\| \leq\|a-b\|$ or $\frac{1}{2}\left\|P(a)-P^{2}(a)\right\| \leq\|P(a)-b\|$.

(iii) Either $\|P(a)-P(b)\| \leq \alpha\|a-P(b)\|+\alpha\|b-P(a)\|+\beta\|a-P(a)\|+\beta\|b-P(b)\|+(1-2 \alpha-$ $2 \beta)\|a-b\|$ or $\left\|P^{2}(a)-P(b)\right\| \leq \alpha\|P(a)-P(b)\|+\alpha\left\|b-P^{2}(a)\right\|+\beta\left\|P(a)-P^{2}(a)\right\|+\beta \| b-$ $P(b)\|+(1-2 \alpha-2 \beta)\| P(a)-b \|$.

Proof. Since $\frac{1}{2}\|a-P(a)\| \leq\|a-P(a)\|$, we have

$$
\begin{aligned}
\left\|P(a)-P^{2}(a)\right\| \leq & \alpha\left\|a-P^{2}(a)\right\|+\alpha\|P(a)-P(a)\|+\beta\|a-P(a)\|+\beta\left\|P(a)-P^{2}(a)\right\|+(1- \\
& 2 \alpha-2 \beta)\|a-P(a)\| \\
= & \alpha\left\|a-P^{2}(a)\right\|+\beta\|a-P(a)\|+\beta\left\|P(a)-P^{2}(a)\right\|+(1-2 \alpha-2 \beta)\|a-P(a)\| \\
\leq & \alpha\left(\|a-P(a)\|+\left\|P(a)-P^{2}(a)\right\|\right)+\beta\|a-P(a)\|+\beta\left\|P(a)-P^{2}(a)\right\|+(1-2 \alpha \\
& -2 \beta)\|a-P(a)\| .
\end{aligned}
$$

It follows that

$$
(1-\alpha-\beta)\left\|P(a)-P^{2}(a)\right\| \leq(1-\alpha-\beta)\|a-P(a)\| .
$$

Since $(1-\alpha-\beta)>0$, we obtain our desired result.

Now, to establish (ii), we assume the contrary, that is,

$$
\frac{1}{2}\|a-P(a)\|>\|a-b\| \text { and } \frac{1}{2}\left\|P(a)-P^{2}(a)\right\|>\|P(a)-b\| .
$$

Using (i),

$$
\begin{aligned}
\|a-P(a)\| & \leq\|a-b\|+\|b-P(a)\| \\
& <\frac{1}{2}\|a-P(a)\|+\frac{1}{2}\left\|P(a)-P^{2}(a)\right\| \\
& =\|a-P(a)\|,
\end{aligned}
$$

this contradiction proves (ii). The condition (iii) directly follows from (ii).

Lemma 5. Let $P$ be a selfmap on a subset $E$ of a Banach space. If $P$ is generalized $(\alpha, \beta)$-non-expansive, then for all $a, b \in E$, we have $\|a-P(b)\| \leq\left(\frac{3+\alpha+\beta}{1-\alpha-\beta}\right)\|a-P(a)\|+\|a-b\|$.

Proof. By Lemma 4(iii), for all $a, b \in E$, either

$$
\begin{aligned}
\|P(a)-P(b)\| \leq & \alpha\|(a)-P(b)\|+\alpha\|b-P(a)\|+\beta\|a-P(a)\|+\beta\|b-P(b)\| \\
& +(1-2 \alpha-2 \beta)\|a-b\|,
\end{aligned}
$$

or

$$
\begin{aligned}
\left\|P^{2}(a)-P(b)\right\| \leq & \alpha\|P(a)-P(b)\|+\alpha\left\|b-P^{2}(a)\right\|+\beta\left\|P(a)-P^{2}(a)\right\|+\beta \| b \\
& -P(a)\|+(1-2 \alpha-2 \beta)\| P(a)-b \|
\end{aligned}
$$


holds. In the first case, we have

$$
\begin{aligned}
\|a-P(b)\| \leq & \|a-P(a)\|+\|P(a)-P(b)\| \\
\leq & \|a-P(a)\|+\alpha\|a-P(b)\|+\alpha\|b-P(a)\|+\beta\|a-P(a)\|+\beta \| b \\
& -P(b)\|+(1-2 \alpha-2 \beta)\| a-b \| \\
\leq & \|a-P(a)\|+\alpha\|a-P(b)\|+\alpha(\|b-a\|+\|a-P(a)\|)+\beta \| a- \\
& P(a)\|+\beta(\|b-a\|+\|a-P(b)\|)+(1-2 \alpha-2 \beta)\| a-b \| .
\end{aligned}
$$

It follows that

$$
\|a-P(b)\| \leq\left(\frac{1+\alpha+\beta}{1-\alpha-\beta}\right)\|a-P(a)\|+\|a-b\| .
$$

In the second case (also using (i)),

$$
\begin{aligned}
\|a-P(b)\| \leq & \|a-P(a)\|+\left\|P(a)-P^{2}(a)\right\|+\left\|P^{2}(a)-P(b)\right\| \\
\leq & 2\|a-P(a)\|+\left\|P^{2}(a)-P(b)\right\| \\
\leq & 2\|a-P(a)\|+\alpha\|P(a)-P(b)\|+\alpha\left\|b-P^{2}(a)\right\|+\beta \| P(a)- \\
& P^{2}(a)\|+\beta\| b-P(b)\|+(1-2 \alpha-2 \beta)\| P(a)-b \| \\
\leq & 2\|a-P(a)\|+\alpha(\|P(a)-a\|+\|a-P(b)\|)+\alpha(\|b-P(a)\| \\
& \left.+\left\|P(a)-P^{2}(a)\right\|\right)+\beta\left\|P(a)-P^{2}(a)\right\|+\beta(\|b-P(a)\|+\| P(a) \\
& -a\|+\| a-P(b) \|)+(1-2 \alpha-2 \beta)(\|P(a)-b\|) \\
\leq & 2\|a-P(a)\|+\alpha(\|P(a)-a\|+\|a-P(b)\|)+\alpha(\|b-P(a)\|+ \\
& \|a-P(a)\|)+\beta\|a-P(a)\|+\beta(\|b-P(a)\|+\|P(b)-a\|+\| \\
& a-P(b) \|)+(1-2 \alpha-2 \beta)(\|P(a)-b\|) .
\end{aligned}
$$

Thus,

$$
\begin{aligned}
(1-\alpha-\beta)\|a-P(b)\| \leq & (2+\alpha+\beta)\|a-P(a)\|+\alpha(\|b-P(a)\|+\| a-P(a \\
& ) \|)+\beta(\|b-P(a)\|+\|P(a)-a\|)+(1-2 \alpha-2 \beta) \\
& (\|P(a)-b\|) \\
= & (2+\alpha+\beta)\|a-P(a)\|+\alpha\|a-P(a)\|+\beta \| P(a)- \\
& a \|+(1-\alpha-\beta)(\|P(a)-b\|) \\
\leq & (2+\alpha+\beta)\|a-P(a)\|+\alpha\|a-P(a)\|+\beta \| P(a)- \\
& a \|+(1-\alpha-\beta)(\|P(a)-a\|+\|a-b\|) .
\end{aligned}
$$

It follows that

$$
(1-\alpha-\beta)\|a-P(b)\| \leq(3+\alpha+\beta)\|a-P(a)\|+(1-\alpha-\beta)(\|a-b\|) .
$$

Since $(1-\alpha-\beta)>0$, we have

$$
\|a-P(b)\| \leq\left(\frac{3+\alpha+\beta}{1-\alpha-\beta}\right)\|a-P(a)\|+\|a-b\| .
$$

Hence, we have obtained the required result in both the cases.

We finish this section by proving the demiclosed principle. 
Lemma 6. Let $P$ be a selfmap on a subset $E$ of a Banach space having Opial's property. If $P$ is generalized $(\alpha, \beta)$-non-expansive, then the following holds:

$$
\left\{a_{m}\right\} \subseteq E, a_{m} \rightarrow w,\left\|a_{m}-P\left(a_{m}\right)\right\| \rightarrow 0 \Longrightarrow P(w)=w
$$

Proof. From Lemma 5, we have

$$
\left\|a_{m}-P(w)\right\| \leq\left(\frac{3+\alpha+\beta}{1-\alpha-\beta}\right)\left\|a_{m}-P\left(a_{m}\right)\right\|+\left\|a_{m}-w\right\| .
$$

It follows that

$$
\liminf _{m \rightarrow \infty}\left\|a_{m}-P(w)\right\| \leq \liminf _{m \rightarrow \infty}\left\|a_{m}-w\right\| .
$$

By Opial's property, we must have $P(w)=w$. Hence, the conclusions can be reached.

\section{Convergence Theorems in Uniformly Convex Banach Spaces}

In this section, we prove some weak and strong convergence results for the newly introduced class of maps in the context of uniformly convex Banach spaces. From now on, the letter $X$ will stand for the uniformly convex Banach space. Now, it is our purpose to prove some weak and strong convergence for the newly introduced class of maps through a faster iterative algorithm. Let $P$ be a selfmap on a closed convex subset $E$ of $X$ and $\mu_{m}, \xi_{m}, \varrho_{m} \in(0,1)$ for all $m \geq 1$. The well known Mann [19], Ishikawa [20], S [21], Noor [22], Abbas [8], Thakur [23], and $K$ [24] iterative algorithms read as follows:

$$
\begin{gathered}
\left\{\begin{array}{l}
a_{1}=a \in E, \\
a_{m+1}=\left(1-\mu_{m}\right) a_{m}+\mu_{m} P\left(a_{m}\right),
\end{array}\right. \\
\left\{\begin{array}{l}
a_{1}=a \in E, \\
b_{m}=\left(1-\xi_{m}\right) a_{m}+\xi_{m} P\left(a_{m}\right), \\
a_{m+1}=\left(1-\mu_{m}\right) a_{m}+\mu_{m} P\left(b_{m}\right),
\end{array}\right. \\
\left\{\begin{array}{l}
a_{1}=a \in E, \\
c_{m}=\left(1-\varrho_{m}\right) a_{m}+\varrho_{m} P\left(a_{m}\right), \\
b_{m}=\left(1-\xi_{m}\right) a_{m}+\xi_{m} P\left(c_{m}\right), \\
a_{m+1}=\left(1-\mu_{m}\right) a_{m}+\mu_{m} P\left(b_{m}\right),
\end{array}\right. \\
\left\{\begin{array}{l}
a_{1}=a \in E, \\
b_{m}=\left(1-\xi_{m}\right) a_{m}+\xi_{m} P\left(a_{m}\right), \\
a_{m+1}=\left(1-\mu_{m}\right) P\left(a_{m}\right)+\mu_{m} P\left(b_{m}\right),
\end{array}\right. \\
\left\{\begin{array}{l}
a_{1}=a \in E, \\
c_{m}=\left(1-\varrho_{m}\right) a_{m}+\varrho_{m} P\left(a_{m}\right), \\
b_{m}=\left(1-\xi_{m}\right) P\left(a_{m}\right)+\xi_{m} P\left(b_{m}\right), \\
a_{m+1}=\left(1-\mu_{m}\right) P\left(b_{m}\right)+\mu_{m} P\left(c_{m}\right),
\end{array}\right. \\
\left\{\begin{array}{l}
a_{1}=a \in E, \\
b_{m}=P\left(\left(1-\mu_{m}\right) a_{m}+\mu_{m} c_{m}\right), \\
a_{m+1}=P\left(b_{m}\right),
\end{array}\right.
\end{gathered}
$$


and

$$
\left\{\begin{array}{l}
a_{1}=a \in E, \\
c_{m}=\left(1-\xi_{n}\right) a_{m}+\xi_{m} P\left(a_{m}\right), \\
b_{m}=P\left(\left(1-\mu_{m}\right) P\left(a_{m}\right)+\mu_{m} P\left(c_{m}\right)\right), \\
a_{m+1}=P\left(b_{m}\right) .
\end{array}\right.
$$

In [21], Agarwal et al. proved that the iterative algorithm (4) is better than the Mann iterative algorithm (1) for contraction maps. In addition, in [8], Abbas and Nazir proved that the iterative algorihm (5) is better than the iterative algorihms (1)-(4) for non-expansive maps. Moreover, in [23], Thakur et al. proved that the iterative algorithm (6) is better than the iterative algorithms (1)-(5) for Suzuki maps. Very recently, in [24], Hussian et al. proved that the iterative algorithm (7) is better than all of the iterative algorithms (1)-(6) for Suzuki maps.

In this article, we present some weak and strong convergence results using $K$ iterative algorithm for the class of genralized $(\alpha, \beta)$-non-expansive maps. Similar results for the algorithms (1)-(6) can be proved on the same line of proofs.

Lemma 7. Let $P$ be selfmap on a closed convex subset $E$ of $X$. If $P$ is a generalized $(\alpha, \beta)$-non-expansive with $F(P) \neq \varnothing$ and $\left\{a_{m}\right\}$ is a sequence generated by the algorithm (7), then $\lim _{m \rightarrow \infty}\left\|a_{m}-z\right\|$ exists for each $z \in F(P)$.

Proof. Let $z \in F(P)$. By Lemma 2, we have

$$
\begin{aligned}
\left\|c_{m}-z\right\| & =\left\|\left(1-\xi_{m}\right) a_{m}+\xi_{m} P\left(a_{m}\right)-z\right\| \\
& \leq\left(1-\xi_{m}\right)\left\|a_{m}-z\right\|+\xi_{m}\left\|P\left(a_{m}\right)-z\right\| \\
& \leq\left(1-\xi_{m}\right)\left\|a_{m}-z\right\|+\xi_{m}\left\|a_{m}-z\right\| \\
& =\left\|a_{m}-z\right\|,
\end{aligned}
$$

which implies that

$$
\begin{aligned}
\left\|a_{m+1}-z\right\| & =\left\|P\left(b_{m}\right)-z\right\| \leq\left\|b_{m}-z\right\| \\
& =\left\|P\left(\left(1-\mu_{m}\right) P\left(a_{m}\right)+\mu_{m} P\left(c_{m}\right)\right)-z\right\| \\
& \leq\left\|\left(1-\mu_{m}\right) P\left(a_{m}\right)+\mu_{m} P\left(c_{m}\right)-z\right\| \\
& \leq\left(1-\mu_{m}\right)\left\|P\left(a_{m}\right)-z\right\|+\mu_{m}\left\|P\left(c_{m}\right)-z\right\| \\
& \leq\left(1-\mu_{m}\right)\left\|a_{m}-z\right\|+\mu_{m}\left\|c_{m}-z\right\| \\
& \leq\left(1-\mu_{m}\right)\left\|a_{m}-z\right\|+\mu_{m}\left\|a_{m}-z\right\| \\
& =\left\|a_{m}-z\right\| .
\end{aligned}
$$

Thus, $\left\{\left\|a_{m}-z\right\|\right\}$ is bounded below and nonincreasing, which implies that $\lim _{m \rightarrow \infty}\left\|a_{m}-z\right\|$ exists for each $z \in F(P)$.

Now, we give the necessary and sufficient condition for the existence of a fixed point of self generalized $(\alpha, \beta)$-non-expansive map on a closed convex subset $E$ of $X$.

Theorem 1. Let $P$ be a selfmap on a closed convex subset $E$ of $X$. If $P$ is a generalized $(\alpha, \beta)$-non-expansive and $\left\{a_{m}\right\}$ is a sequence generated by the algorithm (7), then $F(P) \neq \varnothing$ if and only if $\left\{a_{m}\right\}$ is bounded and $\lim _{m \rightarrow \infty}\left\|P\left(a_{m}\right)-a_{m}\right\|=0$.

Proof. Suppose that $F(P) \neq \varnothing$ and $z \in F(P)$. Then, by Lemma $7, \lim _{m \rightarrow \infty}\left\|a_{m}-z\right\|$ exists and $\left\{a_{m}\right\}$ is bounded. Put

$$
\lim _{m \rightarrow \infty} \| a_{m}-z||=\gamma
$$


By the proof of Lemma 7 together with (8), we have

$$
\limsup _{m \rightarrow \infty}\left\|c_{m}-z\right\| \leq \limsup _{m \rightarrow \infty}\left\|a_{m}-z\right\|=\gamma .
$$

By Lemma 2, we have

$$
\limsup _{m \rightarrow \infty}\left\|P\left(a_{m}\right)-z\right\| \leq \limsup _{m \rightarrow \infty}\left\|a_{m}-z\right\|=\gamma .
$$

Again, by the proof of Lemma 7, we have

$$
|| a_{m+1}-z|| \leq\left(1-\mu_{m}\right)|| a_{m}-z||+\mu_{m}|| c_{m}-z|| .
$$

It follows that

$$
\left\|a_{m+1}-z\right\|-\left\|a_{m}-z\right\| \leq \frac{\left\|a_{m+1}-z\right\|-\left\|a_{m}-z\right\|}{\mu_{m}} \leq\left\|c_{m}-z\right\|-\left\|a_{m}-z\right\| .
$$

Thus, we can get ||$a_{m+1}-z\|\leq\| c_{m}-z \|$. Therefore,

$$
\gamma \leq \liminf _{m \rightarrow \infty}\left\|c_{m}-z\right\|
$$

From (9) and (11), we have

$$
\gamma=\lim _{m \rightarrow \infty}\left\|c_{m}-z\right\|
$$

From (12), we have

$$
\gamma=\lim _{m \rightarrow \infty}\left\|c_{m}-z\right\|=\lim _{m \rightarrow \infty}\left\|\left(1-\xi_{m}\right)\left(a_{m}-z\right)+\xi_{m}\left(P\left(a_{m}\right)-z\right)\right\| .
$$

Since $0<\xi_{m}<1$ for all $m \geq 1$, by Lemma 1 , we have

$$
\lim _{m \rightarrow \infty}\left\|P\left(a_{m}\right)-a_{m}\right\|=0 .
$$

Conversely, we assume that $\left\{a_{m}\right\}$ is bounded and $\lim _{m \rightarrow \infty}\left\|P\left(a_{m}\right)-a_{m}\right\|=0$. Let $z \in A_{c}\left(E,\left\{a_{m}\right\}\right)$. By Lemma 5, we have

$$
\begin{aligned}
A_{r}\left(P(z),\left\{a_{m}\right\}\right) & =\limsup _{m \rightarrow \infty}\left\|a_{m}-P(z)\right\| \\
& \leq\left(\frac{3+\alpha+\beta}{1-\alpha-\beta}\right) \limsup _{m \rightarrow \infty}\left\|P\left(a_{m}\right)-a_{m}\right\|+\limsup _{m \rightarrow \infty}\left\|a_{m}-z\right\| \\
& =\limsup _{m \rightarrow \infty}\left\|a_{m}-z\right\| \\
& =A_{r}\left(z,\left\{a_{m}\right\}\right) .
\end{aligned}
$$

It follows that $P(z) \in A_{c}\left(E,\left\{a_{m}\right\}\right)$. Since $X$ is uniformly convex, we have $A_{c}\left(E,\left\{a_{m}\right\}\right)$ consists of a unique element. Thus, we have $P(z)=z$.

The following result is based on the compactness of the domain.

Theorem 2. Let $P$ be a selfmap on compact convex subset $E$ of $X$. Let $P$ be a generalized $(\alpha, \beta)$-non-expansive with $F(P) \neq \varnothing$, then $\left\{a_{m}\right\}$ generated by the algorithm (7) converges strongly to an element of $F(P)$.

Proof. By Theorem $1, \lim _{m \rightarrow \infty}\left\|P\left(a_{m}\right)-a_{m}\right\|=0$. By compactness assumption, one can find a strongly convergent subsequence namely $\left\{a_{m_{k}}\right\}$ of $\left\{a_{m}\right\}$ such that $a_{m_{k}} \longrightarrow s$ for some $s \in E$. By Lemma 5 , we have

$$
\left\|a_{m_{k}}-P(s)\right\| \leq\left(\frac{3+\alpha+\beta}{1-\alpha-\beta}\right)\left\|a_{m_{k}}-P\left(a_{m_{k}}\right)\right\|+\left\|a_{m_{k}}-s\right\| \longrightarrow 0 .
$$


Since in Banach spaces, convergent sequence has a unique limit, we have $P(s)=s$. By Lemma 7 , $\lim _{m \rightarrow \infty}\left\|a_{m}-s\right\|$ exists. Hence, $s$ is the strong limit of $\left\{a_{m}\right\}$.

We state the following result without the proof because its proof is elementary.

Theorem 3. Let $P$ be a selfmap on a closed convex subset $E$ of $X$. Let $P$ be a generalized $(\alpha, \beta)$-non-expansive and $\left\{a_{m}\right\}$ is a sequence generated by the algorithm (7). If $F(P) \neq \varnothing$ and $\liminf _{m \rightarrow \infty} \operatorname{dist}\left(a_{m}, F(P)\right)=0$, then $\left\{a_{m}\right\}$ converges strongly to an element of $F(P)$.

The next result is based on Condition $I$.

Definition 6. [25] A selfmap $P$ on a subset $E$ of a Banach space is said to satisfy condition I if there is a nondecreasing function $h: \mathbb{R}^{+} \rightarrow \mathbb{R}^{+}$with the property $h(0)=0$ and $h(u)>0$ for all $u \in(0, \infty)$ such that $\|a-P(a)\| \geq h(\operatorname{dist}(a, F(P)))$ for all $a \in E$.

Theorem 4. Let $P$ be a selfmap on a closed convex subset $E$ of $X$. Let $P$ be generalized $(\alpha, \beta)$-non-expansive with $F(P) \neq \varnothing$. If $P$ satisfies condition $I$, then $\left\{a_{m}\right\}$ generated by the algorithm (7) converges strongly to an element of $F(P)$.

Proof. From Theorem 1, it follows that

$$
\liminf _{m \rightarrow \infty}\left\|P\left(a_{m}\right)-a_{m}\right\|=0 .
$$

Since $T$ satisfies condition $I$, we have

$$
\left\|a_{m}-P\left(a_{m}\right)\right\| \geq h\left(\operatorname{dist}\left(a_{m}, F(P)\right)\right) .
$$

From (13), we get

$$
\liminf _{m \rightarrow \infty} h\left(\left(\operatorname{dist}\left(a_{m}, F(P)\right)\right)=0 .\right.
$$

Since the function $h: \mathbb{R}^{+} \rightarrow \mathbb{R}^{+}$is nondecreasing with the property $h(0)=0$ and $h(u)>0$ for each $u \in(0, \infty)$, we have

$$
\liminf _{m \rightarrow \infty} \operatorname{dist}\left(a_{m}, F(P)\right)=0 .
$$

The conclusion follows from Theorem 3.

The following result is based on the Opial condition.

Theorem 5. Let $P$ be a selfmap on a closed convex subset $E$ of $X$ having Opial property. If $P$ is generalized $(\alpha, \beta)$-non-expansive with $F(P) \neq \varnothing$, then, $\left\{a_{m}\right\}$ generated by the algorithm (7) converges weakly to an element of $F(P)$.

Proof. By Theorem 1, $\left\{a_{m}\right\}$ is bounded and $\lim _{m \rightarrow \infty}\left\|P\left(a_{m}\right)-a_{m}\right\|=0$. Since $X$ is uniformly convex, $X$ is reflexive. Hence, one can find a weakly convergent subsequence $\left\{a_{m_{j}}\right\}$ of $\left\{a_{m}\right\}$ with weak limit say $v_{1} \in E$. By Lemma 6, we have $v_{1} \in F(P)$. It is sufficient to show that $v_{1}$ is the weak limit $\left\{a_{m}\right\}$. If $v_{1}$ is not the weak limit of $\left\{a_{m}\right\}$, then one can find another weakly convergent subsequence $\left\{a_{m_{k}}\right\}$ of $\left\{a_{m}\right\}$ with a weak limit, say $v_{2} \in E$ and $v_{2} \neq v_{1}$. Again, by Lemma $6, v_{2} \in F(P)$. By Lemma 7 and Opial condition, we have

$$
\begin{aligned}
\lim _{m \rightarrow \infty}\left\|a_{m}-v_{1}\right\| & =\lim _{j \rightarrow \infty}|| a_{m_{j}}-v_{1}\left\|<\lim _{j \rightarrow \infty}|| a_{m_{j}}-v_{2}\right\| \\
& =\lim _{m \rightarrow \infty}\left\|a_{m}-v_{2}\right\|=\lim _{k \rightarrow \infty}|| a_{m_{k}}-v_{2} \| \\
& <\lim _{k \rightarrow \infty}|| a_{m_{k}}-v_{1}\left\|=\lim _{m \rightarrow \infty}|| a_{m}-v_{1}\right\| .
\end{aligned}
$$


This is a contradiction. Hence, the conclusions can be reached.

\section{Example}

The following example shows that there exist maps which are generalized $(\alpha, \beta)$-non-expansive but neither generalized $\alpha$ non-expansive nor $\beta$-Reich-Suzuki type.

Example 1. Define $P: \mathbb{R}^{+} \rightarrow \mathbb{R}^{+}$by $P(a)=\frac{a}{2}$ if $\frac{1}{2}<a<\infty$ and $P(a)=0$ when $0 \leq a \leq \frac{1}{2}$. We shall prove that $P$ is generalized $\left(\frac{1}{4}, \frac{1}{4}\right)$-non-expansive.

We shall divide the proof into three cases.

(i) If $0 \leq a, b \leq \frac{1}{2}$, then we have

$$
\frac{1}{4}|a-P(b)|+\frac{1}{4}|b-P(a)|+\frac{1}{4}|a-P(a)|+\frac{1}{4}|b-P(b)| \geq 0=|P(a)-P(b)| .
$$

(ii) If $\frac{1}{2}<a, b<\infty$, then we have

$$
\begin{aligned}
\frac{1}{4}|a-P(b)|+\frac{1}{4}|b-P(a)|+\frac{1}{4}|a-P(a)|+\frac{1}{4}|b-P(b)| & =\frac{1}{4}\left|a-\frac{b}{2}\right|+\frac{1}{4}\left|b-\frac{a}{2}\right| \\
& +\frac{1}{4}\left|a-\frac{a}{2}\right|+\frac{1}{4}\left|b-\frac{b}{2}\right| \\
& \geq \frac{1}{4}\left|\frac{3 a}{2}-\frac{3 b}{2}\right|+\frac{1}{4}\left|\frac{a}{2}-\frac{b}{2}\right| \\
& \geq \frac{1}{4}\left|\frac{4 a}{2}-\frac{4 b}{2}\right| \\
& =\frac{1}{2}|a-b| \\
& =|P(a)-P(b)| .
\end{aligned}
$$

(iii) If $\frac{1}{2}<a<\infty$ and $0 \leq b \leq \frac{1}{2}$, then we have

$$
\begin{aligned}
\frac{1}{4}|a-P(b)|+\frac{1}{4}|b-P(a)|+\frac{1}{4}|a-P(a)|+\frac{1}{4}|b-P(b)| & =\frac{1}{4}|a|+\frac{1}{4}\left|b-\frac{a}{2}\right|+\frac{1}{4} \mid a- \\
& \frac{a}{2}\left|+\frac{1}{4}\right| b \mid \\
& =\frac{1}{4}|a|+\frac{1}{4}\left|b-\frac{a}{2}\right|+\frac{1}{4}\left|\frac{a}{2}\right|+\frac{1}{4}|b| \\
& \geq \frac{1}{4}\left|\frac{4 a}{2}\right|=\frac{1}{2}|a|=|P(a)-P(b)| .
\end{aligned}
$$

Hence, $P$ is generalized $\left(\frac{1}{4}, \frac{1}{4}\right)$-non-expansive. However, for $a=\frac{1}{2}$ and $b=\frac{4}{5}$, we have $\frac{1}{2} \mid a-$ $P(a)|<| a-b \mid$. However,

(i). $|P(a)-P(b)|>|a-b|$.

(ii). $|P(a)-P(b)|>\frac{1}{4}|a-P(b)|+\frac{1}{4}|b-P(a)|+\left(1-2\left(\frac{1}{4}\right)\right)|a-b|$.

(iii). $|P(a)-P(b)|>\frac{1}{4}|a-P(a)|+\frac{1}{4}|b-P(b)|+\left(1-2\left(\frac{1}{4}\right)\right)|a-b|$.

Hence, $P$ is neither generalized $\frac{1}{4}$-non-expansive nor $\frac{1}{4}$-Reich-Suzuki type. We obtained the influence of initial point for the $K$ iterative algorithm (7) by $\mu_{m}=0.90, \xi_{m}=0.65, \varrho_{m}=0.90$ in the below table.

Remark 4. In Tables 1 and 2, the items in bold show that the K iterative algorithm (7) converges faster than other algorithms for the class of generalized $(\alpha, \beta)$-non-expansive maps. 
Table 1. Influence of initial points for various iterative algorithms.

\begin{tabular}{cccccccc}
\hline \multicolumn{7}{c}{ Number of Iterations Required to Obtain Fixed Point. } \\
\hline Initial Points & Mann & Ishikawa & Noor & S & Abbas & Thakur & K \\
\hline 5 & 32 & 31 & 30 & 4 & 3 & 2 & $\mathbf{2}$ \\
150 & 40 & 36 & 35 & 7 & 5 & 4 & 3 \\
500 & 43 & 38 & 36 & 9 & 6 & 5 & $\mathbf{4}$ \\
1000 & 45 & 39 & 37 & 9 & 6 & 6 & $\mathbf{4}$ \\
5000 & 48 & 42 & 39 & 11 & 8 & 7 & $\mathbf{5}$ \\
10000 & 50 & 43 & 40 & 12 & 8 & 7 & $\mathbf{6}$ \\
\hline
\end{tabular}

Table 2. Influence of parameters: comparison of various iterative algorithms.

\begin{tabular}{|c|c|c|c|c|c|c|}
\hline \multirow{2}{*}{ Iterations } & \multicolumn{6}{|c|}{ Initial Points } \\
\hline & 10 & $10^{2}$ & $10^{3}$ & $10^{4}$ & $10^{5}$ & $10^{6}$ \\
\hline \multicolumn{7}{|c|}{ For $\mu_{m}=\frac{m}{(m+1)^{\frac{10}{9}}}, \xi_{m}=\frac{1}{(m+3)^{\frac{2}{3}}}, \varrho_{m}=\frac{m}{(3 m+1)}$} \\
\hline Mann & 39 & 45 & 51 & 58 & 64 & 70 \\
\hline Ishikawa & 37 & 43 & 49 & 55 & 60 & 67 \\
\hline Noor & 37 & 42 & 48 & 54 & 60 & 66 \\
\hline S & 5 & 8 & 11 & 14 & 17 & 21 \\
\hline Abbas & 4 & 6 & 9 & 11 & 13 & 16 \\
\hline Thakur & 3 & 5 & 6 & 8 & 9 & 11 \\
\hline K & 2 & 3 & 4 & 5 & 6 & 7 \\
\hline \multicolumn{7}{|c|}{ for $\mu_{m}=\frac{m}{(m+7)^{\frac{17}{14}}}, \xi_{m}=\frac{m}{(m+2)}, \varrho_{m}=m^{-\frac{1}{3}}$} \\
\hline Mann & 95 & 107 & 120 & 133 & 146 & 159 \\
\hline Ishikawa & 89 & 97 & 105 & 113 & 121 & 130 \\
\hline Noor & 88 & 96 & 103 & 111 & 119 & 126 \\
\hline S & 6 & 8 & 11 & 14 & 17 & 19 \\
\hline Abbas & 3 & 5 & 6 & 8 & 10 & 11 \\
\hline Thakur & 3 & 5 & 6 & 8 & 9 & 11 \\
\hline K & 2 & 3 & 4 & 5 & 7 & 8 \\
\hline \multicolumn{7}{|c|}{ for $\mu_{m}=1-\left(\frac{1}{5 m+3}\right)^{\frac{1}{2}}, \xi_{m}=m^{-3}, \varrho_{m}=1-\frac{1}{(2 m+5)}$} \\
\hline Mann & 23 & 26 & 30 & 33 & 37 & 40 \\
\hline Ishikawa & 22 & 25 & 29 & 32 & 36 & 40 \\
\hline Noor & 22 & 25 & 29 & 32 & 36 & 39 \\
\hline S & 5 & 8 & 12 & 15 & 18 & 22 \\
\hline Abbas & 3 & 5 & 7 & 8 & 10 & 12 \\
\hline Thakur & 3 & 4 & 6 & 8 & 9 & 11 \\
\hline K & 2 & 3 & 4 & 5 & 6 & 8 \\
\hline \multicolumn{7}{|c|}{ for $\mu_{m}=\frac{2 m}{(9 m+8)}, \xi_{m}=1-\frac{1}{(m+7)^{2}}, \varrho_{m}=1-\frac{6 m}{(7 m+3)^{4}}$} \\
\hline Mann & 166 & 185 & 205 & 224 & 244 & 265 \\
\hline Ishikawa & 155 & 168 & 181 & 194 & 206 & 219 \\
\hline Noor & 153 & 164 & 174 & 185 & 196 & 206 \\
\hline$S$ & 5 & 8 & 11 & 14 & 17 & 20 \\
\hline Abbas & 3 & 5 & 6 & 8 & 9 & 11 \\
\hline Thakur & 3 & 5 & 6 & 8 & 9 & 11 \\
\hline K & 2 & 3 & 4 & 5 & 6 & 8 \\
\hline \multicolumn{7}{|c|}{ for $\mu_{m}=\frac{m}{(m+5)}, \xi_{m}=\frac{m}{\left(36 m^{2}+1\right)^{\frac{1}{2}}}, \varrho_{m}=\left(\frac{2 m}{(4 m+5)}\right)^{\frac{1}{2}}$} \\
\hline Mann & 32 & 35 & 39 & 42 & 45 & 49 \\
\hline Ishikawa & 31 & 34 & 37 & 40 & 44 & 47 \\
\hline Noor & 31 & 34 & 37 & 40 & 43 & 46 \\
\hline S & 6 & 9 & 12 & 15 & 19 & 22 \\
\hline Abbas & 4 & 5 & 7 & 9 & 11 & 13 \\
\hline Thakur & 3 & 5 & 6 & 8 & 10 & 11 \\
\hline K & 2 & 3 & 4 & 6 & 7 & 8 \\
\hline
\end{tabular}




\section{Conclusions}

In this article, we have presented a new wider class of generalized non-expansive maps, namely, the class of generalized $(\alpha, \beta)$-non-expansive maps. We have also established fundamental properties of these maps in Banach spaces. We have proved that $K$ iterative algorithm of Hussain et al. [24] converges faster to a fixed point of a map in this class. Since the class of generalized $(\alpha, \beta)$-non-expansive maps properly includes the classes of non-expansive, Suzuki non-expansive, Reich-Suzuki type non-expansive, and generalized $\alpha$-non-expansive maps, our results extend the corresponding work proved and discussed in $[1-3,8,11,23,24,26-28]$.

Author Contributions: K.U., J.A. and M.d.1.S. contributed equally to this paper. All authors have read and agreed to the published version of the manuscript.

Funding: This research was funded by Basque Government Grant IT1207-1.

Acknowledgments: The authors are grateful to the Spanish Government for Grant RTI2018-094336-B-I00 (MCIU/AEI/FEDER, UE) and to the Basque Government for Grant IT1207-19.

Conflicts of Interest: The authors declare that they have no conflict of interest.

\section{References}

1. Kirk, W.A. A fixed point theorem for mappings which do not increase distance. Am. Math. Mon. 1965, 72, 1004-1006. [CrossRef]

2. Browder, F.E. Nonexpansive nonlinear operators in a Banach space. Proc. Natl. Acad. Sci. USA 1965, 54, 1041-1044. [CrossRef] [PubMed]

3. Gohde, D. Zum Prinzip der Kontraktiven Abbildung. Math. Nachr. 1965, 30, 251-258. [CrossRef]

4. Byrne, C. Unified treatment of some algorithms insignal processing andimage construction. Inverse Probl. 2004, 20, 103-120. [CrossRef]

5. Podilchuk, C.I.; Mammone, R.J. Image recovery by convex projections using a least squares constraint. J. Opt. Soc. Am. 1990, 7, 517-521. [CrossRef]

6. Youla, D. On deterministic convergence of iterations of related projection mappings. J. Vis. Commun. Image Represent. 1990, 1, 12-20. [CrossRef]

7. Yambangwai, D.; Aunruean, S.; Thianwan, T. A new modified three step iteration method for G-non-expansive mappings in Banach spaces with a graph. Numerical Algor. 2019. [CrossRef]

8. Abbas, M.; Nazir, T. A new faster iteration process applied to constrained minimization and feasibility problems. Math. Vesnik 2014, 66, 223-234.

9. Suzuki, T. Fixed point theorems and convergence theorems for some generalized non-expansive mapping. J. Math. Anal. Appl. 2008, 340, 1088-1095. [CrossRef]

10. Aoyama, K.; Kohsaka, F. Fixed point theorem for $\alpha$-non-expansive mappings in Banach spaces. Nonlinear Anal. 2011, 74, 4387-4391. [CrossRef]

11. Pant, R.; Shukla, R. Approximating fixed points of generalized $\alpha$-non-expansive mappings in Banach spaces. Numer. Funct. Anal. Optim. 2017, 38, 248-266. [CrossRef]

12. Pant, R.; Pandey, R. Existence and convergence results for a class of non-expansive type mappings in hyperbolic spaces. Apllied Gen. Toplogy 2019, 20, 281-295. [CrossRef]

13. Clarkson, J.A. Uniformly convex spaces. Trans. Am. Math. Soc. 1936, 40, 396-414. [CrossRef]

14. Opial, Z. Weak and strong convergence of the sequence of successive approximations for non-expansive mappings. Bull. Am. Math. Soc. 1967, 73, 591-597. [CrossRef]

15. Takahashi, W. Nonlinear Functional Analysis; Yokohoma Publishers: Yokohoma, Japan, 2000.

16. Agarwal, R.P.; O'Regan, D.; Sahu, D.R. Fixed Point Theory for Lipschitzian-Type Mappings with Applications Series; Topological Fixed Point Theory and Its Applications; Springer: New York, NY, USA, 2009; Volume 6.

17. Schu, J. Weak and strong convergence to fixed points of asymptotically non-expansive mappings. Bull. Austral. Math. Soc. 1991, 43, 153-159. [CrossRef]

18. Amini-Harandi, A.; Fakhar, M.; Hajisharifi, H.R. Approximate fixed points of $\alpha$-non-expansive mappings. J. Math. Anal. Appl. 2018, 467, 1168-1173. [CrossRef]

19. Mann, W.R. Mean value methods in iteration. Proc. Am. Math. Soc. 1953, 4, 506-510. [CrossRef] 
20. Ishikawa, S. Fixed points by a new iteration method. Proc. Am. Math. Soc. 1974, 44, 147-150. [CrossRef]

21. Agarwal, R.P.; O'Regon, D.; Sahu, D.R. Iterative construction of fixed points of nearly asymtotically non-expansive mappings. J. Nonlinear Convex Anal. 2007, 8, 61-79.

22. Noor, M.A. New approximation schemes for general variational inequalities. J. Math. Anal. Appl. 2000, 251, 217-229. [CrossRef]

23. Thakur, B.S.; Thakur, D.; Postolache, M. A new iterative scheme for numerical reckoning fixed points of Suzuki's generalized non-expansive mappings. Appl. Math. Comput. 2016, 275, 147-155.

24. Hussain, N.; Ullah, K.; Arshad, M. Fixed point approximation of Suzuki generalized non-expansive mappings via new faster iteration process. J. Nonlinear Convex Anal. 2018, 19, 1383-1393.

25. Senter, H.F.; Dotson, W.G. Approximating fixed points of non-expansive mappings. Proc. Am. Math. Soc. 1974, 44, 375-380. [CrossRef]

26. Khan, S.H.; Fukhar-ud-din, H. Approximating fixed points of $\rho$-non-expansive mappings by RK-iterative process in modular function spaces. J. Nonlinear Var. Anal. 2019, 3, 107-114.

27. Sow, T.M.M. A new general iterative algorithm for solving a variational inequality problem with a quasi-non-expansive mapping in Banach spaces. Commun. Optim. Theory 2019, 2019, 9.

28. Gao, J. The geometry of Banach spaces and fixed points of non-expansive mappings. J. Nonlinear Funct. Anal. 2017, 2017, 10 .

(C) 2020 by the authors. Licensee MDPI, Basel, Switzerland. This article is an open access article distributed under the terms and conditions of the Creative Commons Attribution (CC BY) license (http:/ / creativecommons.org/licenses/by/4.0/). 